\title{
Substitution Permitted
}

National Cancer Institute

\section{Source}

National Cancer Institute. Substitution Permitted. NCI Thesaurus. Code C67005.

A label that specifies whether substitution by a local brand with the same active substance is permitted. 